\title{
Materials selection in mechanical design
}

\author{
M.F. ASHBY and D. CEBON
}

Engineering Design Centre, Engineering Department, Trumpington Street, Cambridge CB2 1PZ, England

\begin{abstract}
A novel materials-selection procedure has been developed and implemented in software. The procedure makes use of Materials Selection Charts: a new way of displaying material property data; and performance indices: combinations of material properties which govern performance. Optimisation methods are employed for simultaneous selection of both material and shape.
\end{abstract}

\section{INTRODUCTION}

The performance of an engineering component is limited by the properties of the material of which it is made, and by the shapes to which this material can be formed. Under some circumstances a material can be selected satisfactorily by specifying ranges for individual properties. More often, however, performance depends on a combination of properties, and then the best material is selected by maximising one or more 'performance indices'. An example is the specific stiffness $E / p$ ( $E$ is Youngs modulus and $p$ is the density). Performance indices are governed by the design objectives. One is derived later in this paper and many others are tabulated elsewhere $[1,2]$. Component shape is also an important consideration. Hollow tubular beams are lighter than solid ones for the same bending stiffness and I-section beams may be better still. Information about section shape can be included in the performance index to enable simultaneous selection of material and shape.

\section{THE PROCEDURE}

\section{Performance Indices}

A performance index is a group of material properties which governs some aspect of the performance of a component $[1,2]$. They are derived from simple models of the function of the component, as illustrated by the following example.

A material is required for a light, stiff beam. The aim is to achieve a specified bending stiffness at minimum weight. The beam has a length $L$ and a square, solid, cross-section as shown in Figure 1a. The mass of the beam is

$$
\mathrm{m}=\mathrm{AL} \rho \text {, }
$$

where $A$ is the area of the cross-section and $\rho$ is the density of the material of which the beam is made. The stiffness $S$ of a simply-supported beam with modulus $E$, second moment of area $I$, central load $F$, and central deflection $\delta$, is

$$
S=\frac{F}{\delta}=\frac{C_{1} E I}{L^{3}} .
$$


with $C_{1}=48$ for 3-point bending. Other supports, or other distributions of load, change $C_{1}$, but nothing else. Assume that the beam has a square section, of side $b$. The second moment of area is

$$
\mathrm{I}=\mathrm{b}^{4} / 12=\mathrm{A}^{2} / 12
$$

The stiffness $S$ and the length $L$ are constrained by the design. The area $A$ is a 'free' variable that we wish to choose so as to minimise the mass, while meeting the constraints.

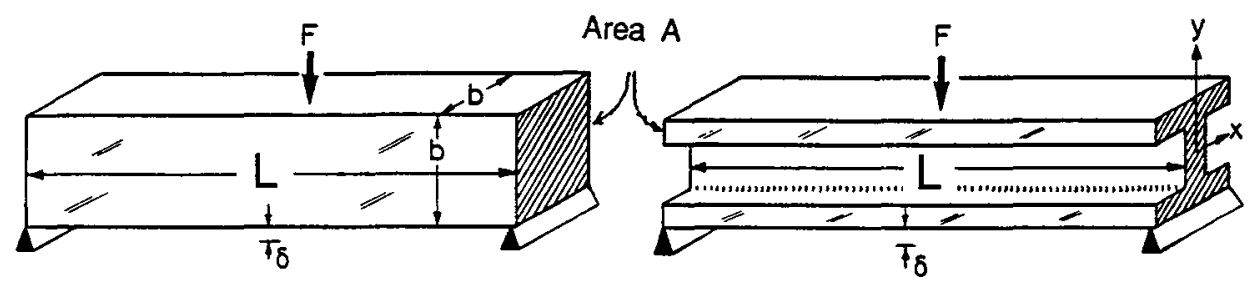

Fig. 1 (a) A square-section beam loaded in bending

(b) A beam of more complex cross section.

Substituting for I in equation (2) and eliminating $\mathrm{A}$ between this and (1) gives

$$
\mathrm{m}=\left(\frac{12 \mathrm{~S}}{\mathrm{C}_{1}}\right)^{1 / 2}(\mathrm{~L})^{5 / 2}\left(\frac{\rho}{\mathrm{E}^{1 / 2}}\right) \text {. }
$$

The mass of the beam can be minimised (and performance maximised) by seeking the material with the largest value of the performance index

$$
M_{1}=\frac{E^{1 / 2}}{\rho} .
$$

The same performance index holds for square-section beams with any value of the design stiffness $S$, any boundary conditions and distributions of load (defined by $C_{1}$ ), and any length $L$.

The cross-section shape of the beam (like the I-section shown in Figure 1b) can be included in the performance index by introducing a dimensionless shape factor $\phi$, defined [3] by

$$
\phi=\frac{4 \pi I}{\mathrm{~A}^{2}}
$$

The value of $\phi$ measures the bending efficiency of the section shape. For the solid section of Figure 1(a), $\phi \cong 1$; that for the I-section of Figure 1(b) is about 5. Real I-sections have efficiencies, $\phi$, as high as 40 . The maximum value of $\phi$ is limited by manufacturing constraints or by local buckling of the component, and, for this reason, it can be considered to be a material property. Shape factors can also be defined for design against yield or fracture, and for shafts as well as beams. Using equation (6) in place of equation (3) to eliminate $\mathrm{A}$ in equation (1) gives the new index:

$$
M_{2}=\frac{(\phi E)^{1 / 2}}{\rho}
$$

For a constant shape ( $\phi$ constant) the criterion reduces to the earlier one; the best selection is then the material with the largest value of $M_{1}$ (equation (5)). In comparing materials with different shapes, the best choice is that with the greatest value of $\mathrm{M}_{2}$ (equation (7)). 


\section{Material Property Charts}

Material selection using performance indices is best achieved by plotting one material property (or mathematical combination of properties) on each axis of a materials selection chart $[1,2]$. In the example shown in Figure 2, the axes are Young's modulus and density. The logarithmic scales span a range so wide that all materials are included. When data for a given material class such as metals are plotted on these axes, it is found that they occupy a field which can be enclosed in a 'balloon'. Ceramics also occupy a field, and so do polymers, elastomers, composites, and so on. The fields may overlap, but are nonetheless distinct. Individual materials or sub-classes (like steels, or polypropylenes, PP) appear as little 'bubbles' which define the ranges of their properties. Hardcopy charts relating many mechanical and thermal properties are now available [1] (two appear in this article). Others can be constructed with the software described in a moment.

The subset of materials with the greatest value of $\mathrm{M}_{1}$ can be identified rapidly by taking $\log$ arithms of equation $(5)\left(\log E=2 \log p+2 \log M_{1}\right)$, and plotting the resulting selection line of slope 2 on the chart. The construction is illustrated in Figure 2, from which it can be seen that woods, fibre reinforced composites and some ceramics are the best choices for a light stiff beam with square cross-section. When section shape is included in the selection criterion, (as in equation (7)) woods become considerably less attractive, because they cannot be manufactured in thin sections with large shape factors, like metals.

SELECTION LINE

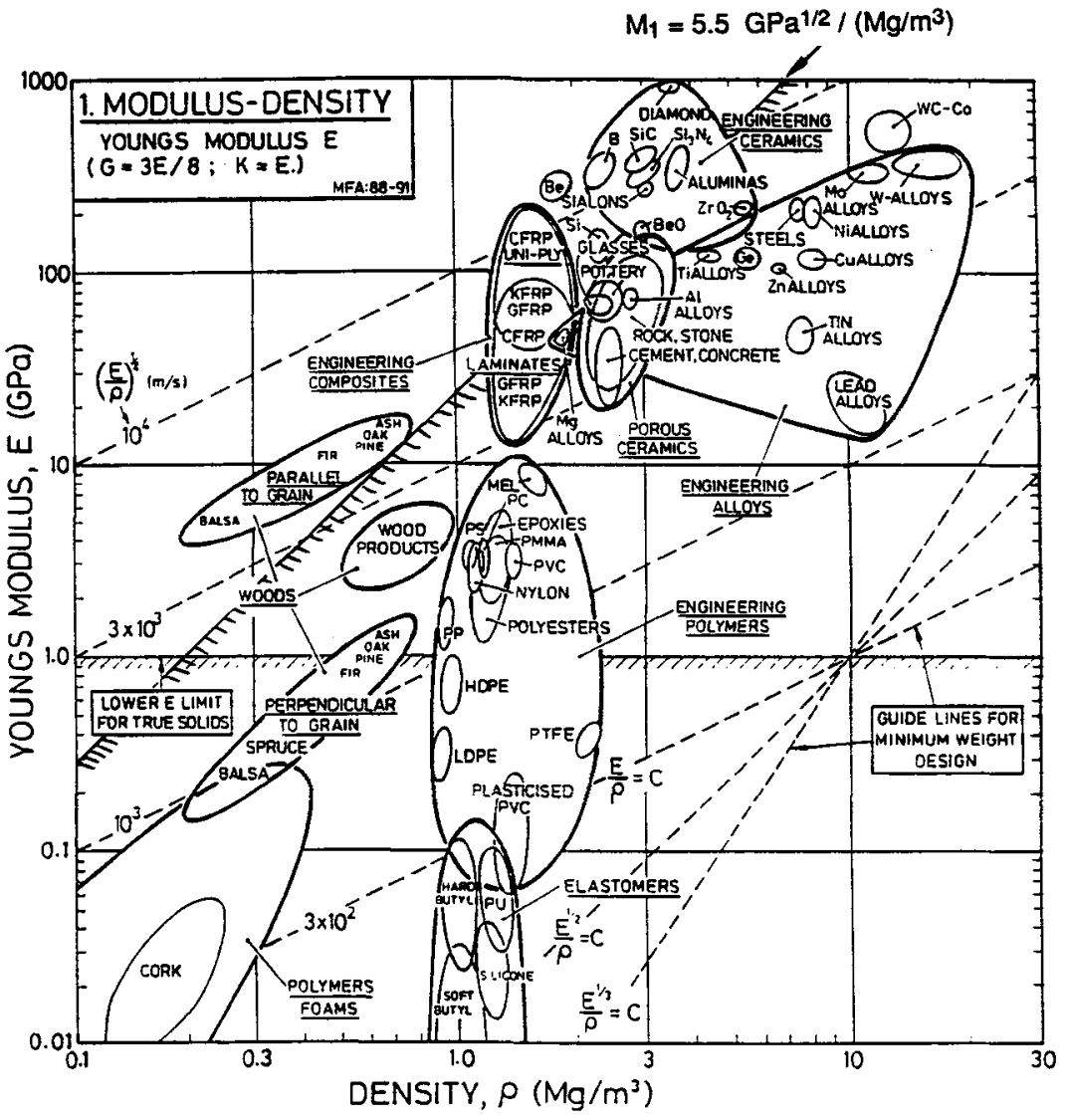

Fig.2 A modulus/density chart illustrating the selection of materials with high values of $M_{1}=E^{1 / 2} / \rho$. Contours of constant $E^{1 / 2 / \rho}$ appear as a family of lines of slope 2 . Materials with $\mathrm{M}_{1}$ greater than a specified value can be identified. 


\section{IMPLEMENTATION IN SOFTWARE}

The 'Cambridge Materials Selector' (CMS) is a computer package consisting of a data base of material properties, a management system which recovers and manipulates the data, and a graphical user interface which presents the property data as material selection charts. The approach employs a number of novel features [4].

\section{The Selection Process}

To select a material, the user performs a series of selection stages. On each stage, a pair of material properties (or user-defined functions of material properties, like $\mathrm{E}^{1 / 2} / \mathrm{\rho}$ ) is specified. The program generates a graph with these properties as the axes. All materials contained in the database with applicable data entries are plotted on the graph. The area of the graph which satisfies the selection criterion is specified by the user, and the materials which lie in that area are considered to have 'passed' the selection stage. Up to six independent selection stages can be performed.

The program stores the results of each selection stage and these can be examined at any time. It is possible to modify any selection stage so that performance criteria can be tightened or relaxed until suitable materials are found. A summary of the $C M S$ session can be stored in a disk file and read into the package later. This enables users to continue/modify a selection where they left-off and to re-evaluate the selection criteria in the light of other design information. It also documents the selection process.

A number of data manipulation routines are available during each selection stage. These include zooming-in on an area of the graph, listing the properties of particular materials and displaying the materials which passed all the previous stages. Facilities are available for plotting hard copies of graphs and listing text information.

\section{Materials, Properties and Data}

Three different types of data are stored in the database: (i) Numerical data, (eg density); (ii) Discrete data (eg material identifier); and (iii) Text data (eg 'typical uses'). Both the numerical and discrete data types can be plotted, giving three types of materials selection charts: (i) Charts with two numerical axes like the hand drawn chart of figure 2; (ii) Charts with one numerical and one discrete axis are 'bar charts'; and (iii) Charts with two discrete properties are 'tables' with each material fitting into one or more cells in the table. The primary, 'generic' CMS database contains Metals, Polymers, Ceramics, Composites and Natural materials. It contains a wide range of mechanical, thermal and electrical properties, as well as information on environmental performance, processing, available forms, typical uses, suppliers, and sources of further information. The package employs an automatic data checking procedure, which also ensures that the database is complete [4]. Future versions of the program will handle additional detailed databases of materials: in particular classes, and for specialised industrial sectors. Several of these databases are in an advanced stage of development at the time of writing.

\section{CASE STUDIES}

\section{Materials for Oars}

Boats, before steam power, could be propelled by poling, by sail and by oar. Oars gave more control than the other two, the military potential of which was well understood by the Romans, the Vikings and the Venetians. But credit for inventing the rowed boat must go to the Egyptians: boats with oars appear in carved relief on monuments built in Egypt between 3300 and $3000 \mathrm{BC}$.

Mechanically speaking, an oar is a beam loaded in bending. It must be strong enough to carry the bending moment exerted by the oarsman without breaking, it must have just the right stiffness to match the rower's own characteristics and give the right "feel", and - very important it must be as light as possible. Meeting the strength constraint is easy. Oars are designed on stiffness, that is, to give a specified elastic deflection under a given load. The upper part of 

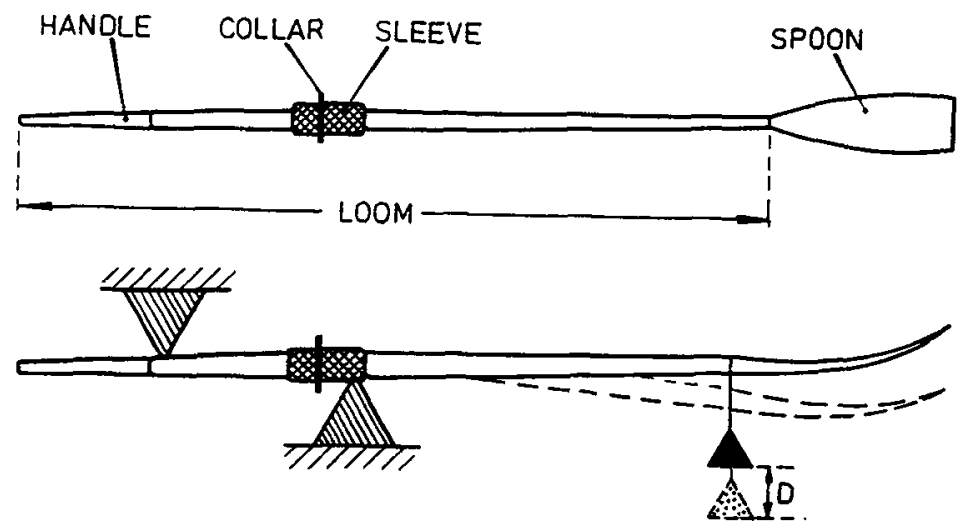

Fig. 3 An oar, showing the components and the method of measuring the stiffness.

Figure 3 shows an oar: a blade or "spoon" is bonded to a shaft or "loom" which carries a sleeve and collar to give positive location in the rowlock. The lower part of the figure shows how the oar stiffness is measured: a $10 \mathrm{~kg}$ weight is hung on the oar $2.05 \mathrm{~m}$ from the collar and the deflection at this point is measured. A soft oar will deflect nearly $50 \mathrm{~mm}$; a hard one only 30 . A rower, when ordering an oar, specifies how hard it should be [5].

The oar must also be light; extra weight increases the wetted area of the hull and the drag that goes with it. So there we have it: an oar is a beam of specified stiffness and minimum weight. The performance index we want was derived in earlier; it is

$$
M_{1}=\frac{E^{1 / 2}}{\rho} \text {. }
$$

What materials make good oars? Figure 2 shows the appropriate chart, with a selection line for the index placed on it. It identifies three classes of material: woods, carbon- and glassfibre reinforced polymers and certain ceramics (Table 1). Ceramics are brittle; they have low values of toughness; if you dropped a ceramic oar, it would probably shatter. This can be analysed, leading to further performance indices [1], but there is insufficient space to do so here. We simply note that ceramics are eliminated because they are brittle and expensive. The recommendation is clear. Make your oars out of wood or - better - out of CFRP.

\section{TABLE 1 MATERIALS FOR OARS}

\begin{tabular}{|l|l|l|}
\hline MATERIAL & $\mathrm{M}(\mathrm{GPa})^{1 / 2} /\left(\mathrm{Mg} / \mathrm{m}^{3}\right)$ & COMMENT \\
\hline Woods & $5-8$ & Cheap, traditional, but not easily controlled. \\
CFRP & $4-8$ & As good as wood, more control of properties. \\
GFRP & $3.5-5.5$ & Cheaper than CFRP but lower M. \\
Ceramics & $4-8$ & Good $M$ but brittle and expensive \\
\hline
\end{tabular}


Of what, in reality, are oars made? Racing oars and sculls are made either of wood or of a high performance composite: carbon-fibre reinforced epoxy, CFRP. Wooden oars are made today, as they were 100 years ago, by handcraftsmen who use Sitka spruce from the northern US or Canada, the further north the better because the short growing season gives a finer grain. A spruce oar weighs between 4 and $4.3 \mathrm{~kg}$, and costs (in 1993) about $£ 150$ or $\$ 250$. Composite blades are a little lighter than wood, for the same stiffness. The component parts are fabricated from a mixture of carbon and glass fibres in an epoxy matrix, assembled and glued. The advantage of composites lies partly in the saving of weight (typical weight: $3.9 \mathrm{~kg}$ ) and partly in the greater control of performance: the shaft is moulded to give the stiffness specified by the purchaser. At a price, of course: a CFRP oar costs about $£ 300(\$ 450)$.

\section{Materials for Precision Instruments}

The precision of a measuring device, like a sub-micrometer displacement gauge, is limited by its stiffness, and by the dimensional change caused by temperature gradients. Compensation for elastic deflection can be arranged; and corrections to cope with thermal expansion are possible too - provided the device is at a uniform temperature. Thermal gradients are the real problem: they cause a change of shape - that is, distortion - of the device for which compensation is not possible. Sensitivity to vibration is also a problem: natural excitation introduces noise into the measurement. So - in precision instrument design - it is permissible to allow expansion, provided distortion does not occur [6]. Elastic deflection is allowed, provided natural vibration frequencies are high.

What, then, are good materials for precision devices?

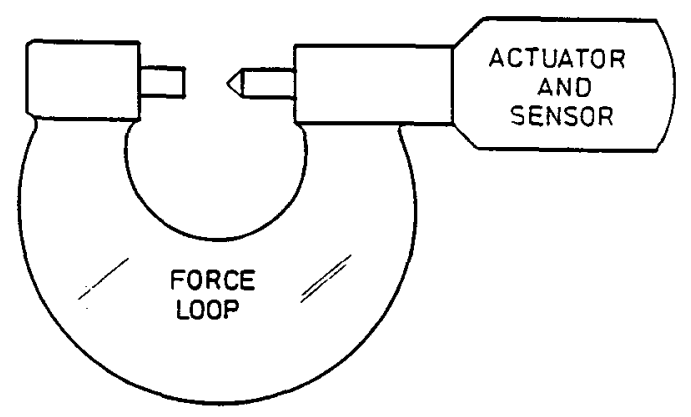

Figure 4. A precision instrument. It consists of a force loop, an actuator and a sensor

Figure 4 shows, schematically, such a device: it consists of a force loop, an actuator and a sensor; we aim to choose a material for the force loop. It will, in general, support heat sources: electrical components which generate heat. The relevant performance index is found by considering the simple case of one-dimensional heat flow through a rod insulated except at its ends, one of which is at ambient and the other connected to the heat source. In the steady state, Fourier's law is

$$
q=-\lambda \frac{d T}{d x}
$$

where $q$ is heat input per unit area, $\lambda$ is the thermal conductivity and $\frac{d T}{d x}$ is the resulting temperature gradient. The strain is related to temperature by 


$$
\varepsilon=\alpha\left(T_{0}-T\right)
$$

where $\alpha$ is the thermal conductivity and $T_{0}$ is ambient temperature, from which

$$
\frac{\mathrm{d} \varepsilon}{\mathrm{dx}}=\frac{\alpha \mathrm{dT}}{\mathrm{dx}}=\left(\frac{\alpha}{\lambda}\right) \mathrm{q}
$$

Thus for a given geometry and heat flow, the distortion $\mathrm{d} \varepsilon / \mathrm{dx}$ is minimised by selecting materials with large values of the index

$$
\mathrm{M}_{3}=\frac{\lambda}{\alpha}
$$

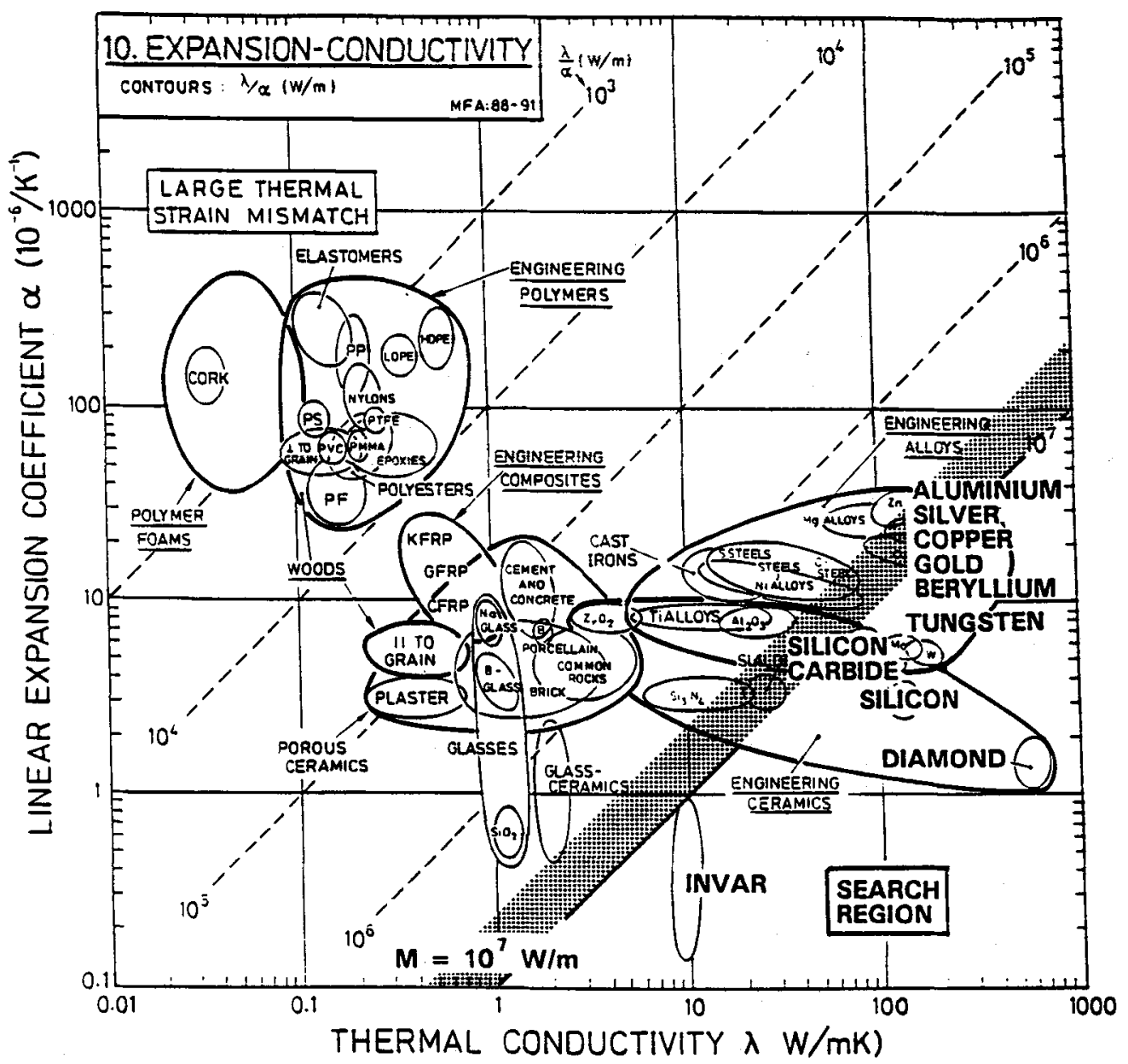

Figure 5. A Chart of thermal conductivity, $\lambda$, and expansion coefficient, $\alpha$, allowing selection of materials for the force loop of precision instruments. 
The other problem is vibration. The sensitivity to external excitation is minimised by making the natural frequencies of the device as high as possible. The flexural vibrations have the lowest frequencies; they are proportional, once again, to

$$
M_{1}=\frac{E^{1 / 2}}{\rho}
$$

A high value of this index will minimise the problem. Finally, of course, the device must not cost too much.

Chart 10 (Figure 5) shows the expansion coefficient, $\alpha$, plotted against the thermal conductivity, $\lambda$. Contours show constant values of the quantity $\lambda / \alpha$. A search region is isolated by the line $\lambda / \alpha=10^{7} \mathrm{~W} / \mathrm{m}$, giving the short list of Table 2 . Values of $M_{1}=E^{1 / 2} / \rho$ read from Chart 1 (Figure 2) are included in the table.

TABLE 2 MATERIALS TO MINIMISE THERMAL DISTORTION

\begin{tabular}{|c|c|c|c|}
\hline MATERIAL & $\begin{array}{c}M_{3}=\lambda / \alpha \\
(W / m)\end{array}$ & $\begin{array}{c}\mathrm{M}_{1}=\mathrm{E}^{1 / 2} / \rho \\
\left(\mathrm{GPa}^{1 / 2} /\left(\mathrm{Mg} / \mathrm{m}^{3}\right)\right)\end{array}$ & COMMENT \\
\hline DIAMOND & $5 \times 10^{8}$ & 8.6 & $\begin{array}{l}\text { Outstanding } \\
\text { expensive. }\end{array}$ \\
\hline SILICON & $4 \times 10^{7}$ & 6.0 & Excellent $M_{1}$ and $M_{3}$; cheap. \\
\hline SILICON CARBIDE & $2 \times 10^{7}$ & 6.2 & $\begin{array}{l}\text { Excellent } M_{1} \text { and } M_{3} \text {; potentially } \\
\text { cheap. }\end{array}$ \\
\hline BERYLLIUM & $10^{7}$ & 9 & Less good than silicon or $\mathrm{SiC}$. \\
\hline ALUMINIUM & $10^{7}$ & 3.1 & Poor $M_{1}$, but very cheap. \\
\hline SILVER & $2 \times 10^{7}$ & 1.0 & ) High density \\
\hline COPPER & $2 \times 10^{7}$ & 1.3 & gives poor \\
\hline GOLD & $2 \times 10^{7}$ & 0.6 & ) value of $M_{1}$ \\
\hline TUNGSTEN & $3 \times 10^{7}$ & 1.1 & ) Better than copper, silver or \\
\hline MOLYBDENUM & $2 \times 10^{7}$ & 1.3 & ) gold, but less good than \\
\hline INVAR & $3 \times 10^{7}$ & 1.4 & ) silicon, $\mathrm{SiC}$, diamond \\
\hline
\end{tabular}


Diamond is outstanding, but practical only for very small devices. The metals, except for beryllium, are disadvantaged by having high densities and thus poor values of $M_{1}=E^{1 / 2} / \rho$. The best choice is silicon, available in large sections, with high purity. Silicon carbide is an alternative.

Nano-scale measuring and imaging systems present the problem analysed here. The atomic-force microscope and the scanning-tunnelling microscope both support a probe on a force loop, typically with a piezo-electric actuator and electronics to sense the proximity of the probe to the test surface. Closer to home, the mechanism of a video recorder and that of a hard disk drive qualify as precision instruments; both have an actuator moving a sensor (the read head) attached, with associated electronics, to a force loop. The materials identified in this case study are the best choice for force loop.

\section{CONCLUSIONS}

A novel materials selection procedure has been implemented in software. It contains a database of quantitative and qualitative data for a wide range of engineering materials: metals, polymers, ceramics, composites and natural materials. The management system provides an interactive graphical selection environment suitable for mechanical engineering design, employing performance indices for selecting materials with optimum properties and section shape.

\section{ACKNOWLEDGEMENTS}

The authors are very grateful to the Leverhulme Trust for funding development of the Cambridge Materials Selector, and to Ruth Thomas for her assistance with development of the user interface.

\section{REFERENCES}

[1] Ashby, MF. 'Materials selection in mechanical design' Pergamon Press, Oxford, 1992.

[2] Ashby, MF. 'On the engineering properties of materials' Acta Metallurgica, 37, p1273, 1989.

[3] Ashby, MF. 'Materials and shape' Acta Metallurgica, 39, 1025, 1991.

[4] Cebon, D and Ashby, MF. 'Computer-based materials selection for mechanical design' in 'Computerization and networking of materials databases', ASTM STP 1140, American Society for Testing and Materials, Philadelphia, 1992.

[5] Redgrave, S "Complete Book of Rowing", Partridge Press, London (1992).

[6] Chetwynd, D.G. (1987) Precision Engineering, 9, (1), 3. 